\title{
COVID-19 challenges in clinical trials
}

Sir,

Clinical research involves working with human subjects to answer questions relevant to their well-being in an ethical manner. ${ }^{1}$ The current scenario from the past one year has drastically changed the face of the clinical trials. $^{2}$ The present COVID prevalence and simultaneously conducting the research with all the regulations and the precautions has been the difficult task for the contract research organisations (CRO) ${ }^{3-5}$

Delays in patient enrolment or missed study visits resulting from the inability of patients to travel to onsite visits or limitations on nonessential in-office visits or testing and also delay in study initiation activities resulting from the inability to perform site selection/initiation visits and/or clinical vendor qualification visits, and subsequent downstream delays in patient enrolment. ${ }^{6,7}$ Protocol adherence issues arising from an inability to comply with visit schedules, study procedures, drug administration, and monitoring procedures. Delays in clinical material distribution and import/export delays due to limited manufacturing and operations staff, limited or reprioritized hospitals staff, and travel bans. ${ }^{6}$ Some delays in local IRB reviews and approvals due to limited or reprioritized site staff. Cancellation or postponement (indefinitely, in some cases) of major scientific and professional conferences and meetings, and KOL, investigator, and scientific advisory board meetings. The FDA recently issued a guidance, conduct of clinical trials of medicinal products during COVID-19 Pandemic emphasizing mitigation and precautionary strategies based on study-specific circumstances. ${ }^{3}$ It also provides recommendations on the management of procedural modifications, protocol amendments, and deviations (e.g., implementation, IRB approvals, FDA consultation), as well as preparedness to describe the tactics and impact in the corresponding clinical study reports. ${ }^{5,8,9}$

\section{REFERENCES}

1. Current clinical trials. Available at: https:// clinicaltrials.gov/ct2/resources/trends. Accessed on 20 February 2021.

2. DeVito NJ, Bacon S, Goldacre B. Compliance with legal requirement to report clinical trial results on ClinicalTrials.gov: a cohort study. Lancet. 2020;395 (10221):361-9.
3. FDA guidance on conduct of clinical trials of medical products during COVID-19 public health emergency. Available at: https://www.fda.gov/media/136238/ download. Accessed on 20 February 2021.

4. Cinical trial reporting. Available at: https://www.thelancet.com/pdfs/journals/lancet/PIIS0 140-6736(20)32264-9.pdf. Accessed on 20 February 2021.

5. Public disclosure of clinical trial results. Available at: http://www.who.int/ictrp/results/WHO_

Statement_results_reporting_clinical_trials.pdf. Accessed on 20 February 2021.

6. Anderson ML, Chiswell K, Peterson ED, Tasneem A, Topping J, Califf RM. Compliance with results reporting at ClinicalTrials.gov. N Engl J Med. 2015; 372:1031-9.

7. World medical association. World medical association declaration of Helsinki: ethical principles for medical research involving human subjects. JAMA. 2013;310:2191-4.

8. Bagiella E, Bhatt DL, Gaudino M. The consequences of the COVID-19 pandemic on non-COVID-19 clinical trials. J Am Coll Cardiol. 2020;76:342-5.

9. Coronavirus in the US: latest map and case count. Available at: https://www.nytimes.com/interactiv e/2020/us/coronavirus-us-cases.html?action=click \&pgtype $=$ Article \&state $=$ default $\&$ module $=$ styln - coron avirus\&variant $=$ show\&region $=$ TOP_BANNE $\mathrm{R} \&$ context=storylines_menu\#states. Accessed on 13 May 2020

\section{Murugan Panchatcharam,} Sravanthi Lakshmi Mukku*

Amaris Clinical; Division of Caplin Point Laboratories Limited, Chennai, Tamil Nadu, India

*Correspondence to

Dr. Sravanthi Lakshmi Mukku, E-mail: dr.sravanthilakshmimukku@gmail.com

Cite this article as: Panchatcharam M, Mukku SL. COVID-19 challenges in clinical trials. Int J Basic Clin Pharmacol 2021;10:769. 